\title{
ABSOLUTE BUNCH LENGTH MEASUREMENTS AT THE ALS BY INCOHERENT SYNCHROTRON RADIATION FLUCTUATION ANALYSIS*
}

\author{
F. Sannibale, M. S. Zolotorev, LBNL, Berkeley, CA 94720, U.S.A. \\ D. Filippetto INFN-LNF, Frascati, Rome, Italy. \\ G. V. Stupakov, SLAC, Stanford, CA 94309, U.S.A.
}

\begin{abstract}
By analysing the pulse to pulse intensity fluctuations of the radiation emitted by a charge particle in the incoherent part of the spectrum, it is possible to extract information about the spatial distribution of the beam. At the Advanced Light Source (ALS) of the Lawrence Berkeley National Laboratory, we have developed and tested a simple scheme based on this principle that allows for the absolute measurement of the bunch length. A description of the method and the experimental results are presented.
\end{abstract}

\section{INTRODUCTION}

Several different processes allow for the emission of photons from moving charged particles: synchrotron radiation, Cerenkov radiation, transition radiation, etc. In all these processes the presence of incoherent radiation is due to the fact that particles are randomly distributed along the beam. For example, in the case of an ideal coasting beam composed by a large number of particles equally separated by a longitudinal distance $d$ and moving along a circular trajectory, there is no synchrotron radiation emission up to those frequencies whith wavelength $<d$. In fact below those frequencies, the interference between the radiation emitted by the evenly distributed electrons will produce a vanishing net electric field. In a more realistic coasting beam, the same particles are now randomly distributed along the orbit causing a small modulation of the beam current. The effect of such a random modulation is that the interference is not fully destructive anymore and a net nonzero electric field shows up. If the turn by turn position of the particles along the beam changes (due for instance, to longitudinal dispersion or to path length dependence on transverse position), then the modulation changes and the energy radiated in a single pass fluctuates turn by turn. By measuring the radiation intensity over multiple passages, we find that for a sufficiently large sample, the measured spectrum converges to the characteristic incoherent spectrum of the radiation process under observation (synchrotron radiation in our example). The passage from the coasting to the bunched beam case introduces a strong coherent emission at those wavelengths comparable or longer than the bunch length, but does not modify the higher frequency part of the spectrum.

It has been shown for the case of a bunched beam [1], that by measuring the pulse to pulse intensity fluctuation of the radiation within a bandwidth $\Delta \omega$ in a region of the spectrum where no coherent emission is present, it is possible to perform absolute measurements of the bunch length. A frequency domain version of such a technique has been already proved experimentally by using a configuration that requires a photon spectrometer [2-4].

In this paper, we describe a significantly simplified scheme that does not require complex and expensive intrumentation and that allows for accurate absolute measurements of the bunch length. We tested the technique at the ALS and the results were compared with the ones from streak camera measurements.

\section{METHOD DESCRIPTION}

In this scheme, the energy radiated by the bunch in a single pass is measured. The photons are collected within a narrow band around a frequency where no coherent emisssion is present. Values of radiated energy per passage $W$ are measured and recorded for many passages of the beam. $W$ fluctuates passage to passage and its relative rms variation of $\delta$, is given by [1]:

$$
\delta^{2}=\frac{\sigma_{W}^{2}}{\langle W\rangle^{2}}=\int_{-\infty-\infty}^{\infty} \int_{-\infty}^{\infty} d t d t^{\prime} \frac{\left|K\left(t-t^{\prime}\right)\right|^{2}}{|K(0)|^{2}} I(t) I\left(t^{\prime}\right)
$$

whith $I$ the normalized longitudinal bunch distribution and $K$ the time domain response of the system to a delta function excitation (if a bandpass filter is used, $K$ is the inverse Fourier transform of the filter transmission curve).

For the case of a gaussian beam with rms length in time units $\sigma_{\tau}$, and of a gaussian filter with rms bandwidth $\sigma_{c h}$ Eqn. (1) can be readily integrated:

$$
\delta^{2}=1 / \sqrt{1+4 \sigma_{\tau}^{2} \sigma_{\omega}^{2}}
$$

This last expression shows that if $\sigma_{\omega}$ is known, then by measuring $\delta$ one can derive the absolute value of the rms bunch length. Expression (2) has been obtained for gaussian beams, but numerical integration of (1) for nongaussian distributions showed that the accuracy of (2) is good at the few percent level for most of the distributions, as long as they are represented by their rms length and do not include microstructures with characterisitc length much smaller than the bunch length.

For $\sigma_{\tau} \gg 1 /\left(2 \sigma_{\omega}\right)$, we have $\delta^{2} \sim 1 /\left(2 \sigma_{\tau} \sigma_{\omega}\right)$, and using the fact that the longitudinal coherence length for an 
electromagnetic mode with frequency content $\sigma_{\omega}$ is $\sigma_{t c}=1 /\left(2 \sigma_{\omega}\right)^{* *}$ we can write:

$$
\delta^{2}=\sigma_{t c} / \sigma_{\tau}=1 / M
$$

where $M$ is the number of modes contained in the bunch. Equation (3) leads to the nice physical interpretation that the intensity fluctuation is due to $M$ independent modes radiating randomly within the bunch.

The results presented so far assumed a bunch with no transverse size. By including the effect of the finite transverse size for the example case of gaussian transverse distributions we obtain:

$$
\delta^{2}=\left(1+\sigma_{\tau}^{2} / \sigma_{i c}^{2}\right)^{-\frac{1}{2}}\left(1+\sigma_{x}^{2} / \sigma_{x c}^{2}\right)^{-\frac{1}{2}}\left(1+\sigma_{y}^{2} / \sigma_{y c}^{2}\right)^{-\frac{1}{2}}
$$

with $\sigma_{x}$ and $\sigma_{y}$ the rms horizontal and vertical beam sizes respectively and $\sigma_{x c}$ and $\sigma_{y c}$ the coherence sizes of the related transverse electromagnetic modes at the wavelength of operation. Such quantities are defined by the properties of the radiation process and must include diffraction effects due to limiting apertures.

It can be shown that the statistical error on $\delta^{2}$ when $N_{s}$ samples are collected is given by:

$$
\sigma_{\delta^{2}} / \delta^{2}=\sqrt{2 / N_{s}}
$$

\section{EXPERIMENTAL SETUP}

Figure 1, shows the experimental setup for the measurements performed at the beamline BL7.2 of the Advanced Light Source (ALS) in Berkeley. Such a beamline collects the synchrotron radiation from a dipole magnet and has a total angular acceptance of $5.5 \mathrm{mrad}$ and $2.8 \mathrm{mrad}$ for the horizontal and vertical plane respectively (represented by the limiting aperture in Fig. 1). BL7.2 spectrum ranges from the far-infrared up to the top part of the visible portion.

In our application we used visible light, which is in the incoherent part of the spectrum and allows using the large variety and relatively inexpensive optical components and detectors readily available for such a frequency range. A $1 \mathrm{~m}$ focal length lens was used to focus, through a flat mirror, the beam on the photocathode plane of a streak camera (Hamamatsu C5680). The mirror could be retracted in order to allow for the light to go into another branch for the fluctuation measurement. An interferometric filter (Melles Griot) with gaussian transmission curve centered at $632.8 \mathrm{~nm}$ and with peak transmission of $55 \%$ selected the photons within a bandwidth of $1 \mathrm{~nm}$ FWHM. The first lens focal length was chosen for keeping the angle between the incoming photon trajectories and the normal to the filter plane small enough to avoid broadening of the filter bandwidth. Downstream the filter the transmitted photons were finally focused by a microscope objective (Edmund DIN

\footnotetext{
** For a gaussian pulse the uncertainty principle requires $\sigma_{\tau} \sigma_{\omega}=1 / 2$.
}

$10, \mathrm{~F} 0.25$ ) on the $0.2 \mathrm{~mm}^{2}$ sensitive area of an avalanche photodiode (Perkin Elmer C30902S, gain $250, \sim 600 \mathrm{MHz}$ bandwidth, $\sim 60 \%$ quantum efficiency at $632.8 \mathrm{~nm}$ ). The signal from the photodiode was amplified (Hamamatsu C5594, 50k-1.5G Hz bandwidth, $36 \mathrm{~dB}$ gain) and sent to a digital oscilloscope (LeCroy Wavepro $7300 \mathrm{~A}, 3 \mathrm{GHz}$ bandwidth and $20 \mathrm{Gsamples} / \mathrm{sec}$ ) for data recording and analysis. The oscilloscope was triggered with the $\sim 1.5 \mathrm{MHz}$ revolution clock of the ALS.

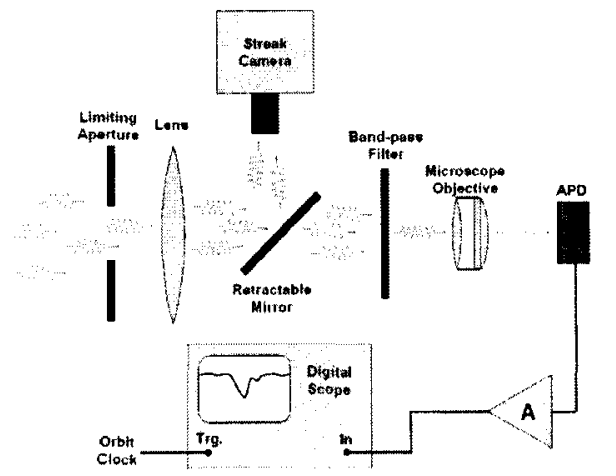

Fig. 1. Schematic diagram of the experimental set-up used for the measurement at BL 7.2.

Figure 2, shows the typical signal visible at the scope when measuring the light from a single passage of a single ALS bunch. The typical rms length of the electron beam is $\sim 25$ ps so that the shape of the pulse in Fig. 2 was totally defined by the response of the measurement system. The oscilloscope was set in order to measure the areas $S_{A B}$ of the signal between points $A$ and $B$, and $S_{C D}$ between points $C$ and $D$ in Fig. 2. $S_{A B}$ is proportional to the number of photons impinging on the detector plus the contribution due to the noise in the signal, while $\mathrm{S}_{\mathrm{CD}}$ is a measure of this noise contribution. The lengths of the segments $A B$ and $C D$ were set to be the same.

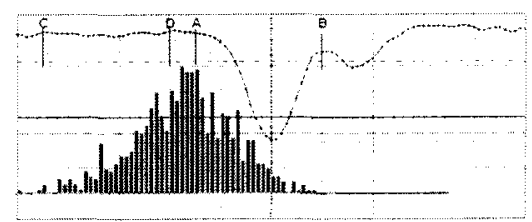

Figure 2. Oscilloscope window showing the track of a typical signal from the photodetector and the histogram of the measured values of the signal area between points $A$ and $\mathrm{B}$. The horizontal scale is $500 \mathrm{ps} / \mathrm{div}$ while the vertical is $50 \mathrm{mV} / \mathrm{div}$.

The scope was also set to calculate the average values for such areas and their standard deviations over 5000 samples per each bunch length measurement $(\sim 1 \mathrm{~min}$ per measurement) in order to keep according to (5), the statistical error at $\sim 2 \%$. In this configuration, $\langle W\rangle$ the average energy radiated per passage by the electron beam is proportional to $\left\langle\mathrm{S}_{\mathrm{AB}}\right\rangle-\left\langle\mathrm{S}_{\mathrm{CD}}\right\rangle$, while its variance $\sigma_{W}{ }^{2}$ is proportional to ${\sigma_{S A B}}^{2}-\sigma_{S C D}{ }^{2}$. By comparing different amplitude signals, we also verified that the shape of the signal itself did not depend on the amplitude. 


\section{EXPERIMENTAL RESULTS}

Simulations and analytical calculations showed that in our configuration, the diffraction due to the finite beamline acceptance fully defines the shape for both the transverse modes, which can be well described by the $[\sin (\xi) / \xi]^{2}$ function typical of a plane wave diffracting through a finite aperture. Nevertheless, numerical calculations also showed that it is still possible to use the gaussian formula (4) if one fits the central peak of the $[\sin (\xi) / \xi]^{2}$ function with a gaussian, and uses for the coherence length the rms width of the fit divided by $\sqrt{2}$. For $\lambda=632.8 \mathrm{~nm}$ and for the acceptances of BL7.2 we obtained $\sigma_{x c}=29.8 \mu \mathrm{m}$ and $\sigma_{y c}=59.5 \mu \mathrm{m}$. The ALS measurements were performed at the two beam energies of 1.2 and $1.9 \mathrm{GeV}$. The rms beam sizes at BL7.2 source point were $\sigma_{x}=64.8 \mu \mathrm{m}$ and $\sigma_{y}=6.3 \mu \mathrm{m}$ at $1.2 \mathrm{GeV}$ and $\sigma_{x}=103.0 \mu \mathrm{m}$ and $\sigma_{y}=10.0 \mu \mathrm{m}$ at $1.9 \mathrm{GeV}$.

Equation (4) was derived according to classical field theory, the proper quantum treatment requires the addition of the shot noise term $1 / N$, with $N$ the number of photons impinging on the detector. Additionally, photodiodes, avalanche photodiodes and photomultipliers all exploit stochastic phenomena for the photon-electron conversion and amplification. This must be accounted by using a modified shot noise term $\kappa^{2}=\zeta /(\eta N)$, where $\zeta$ is the excess noise factor, a constant $\geq 1$ related to the avalanche process and $\eta$ is the detector quantum efficiency. Putting all the contributions together and indicating with $\delta_{M}{ }^{2}$ the measured fluctuation variance one finally obtains for the rms length of the bunch:

$$
\sigma_{\tau}^{2}=\frac{1}{4 \sigma_{\omega}^{2}}\left[\left(\delta_{M}^{2}-\kappa^{2}\right)^{-2}\left(1+\frac{\sigma_{x}^{2}}{\sigma_{x c}^{2}}\right)^{-1}\left(1+\frac{\sigma_{y}^{2}}{\sigma_{y c}^{2}}\right)^{-1}-1\right](6)
$$

The $\kappa^{2}$ term can be measured by performing 2 or more measurements of $\delta_{M}{ }^{2}$ for the same bunch length for different number of photons impinging on the detector (using neutral density filters for instance). All the terms on both sides of (6) remain the same with the exception of the shot noise one. From that, and considering that $<N>\propto\left(<S_{A B}>-<S_{C D}>\right), \kappa^{2}$ can be evaluated.

Figure 3 shows 2 examples of measurements performed for different beam conditions. The agreement with streakcamera data acquired right after each fluctuation measurament is remarkably good especially considering that no parameter has been adjusted to match the data.

The typical rms difference between the streak camera and the fluctuation data was $\sim 4 \%$. This is larger than the $2 \%$ contribution due to (5). The extra error is probably associated with the shot noise term that in our measurements was comparable to $\delta^{2}$.

\section{POSSIBLE UPGRADES \& CONCLUSIONS}

By splitting the light from the source in two paths and using for each of them a bandpass filter with different central wavelength but same bandwidth, it is possible to discriminate between the transverse and longitudinal components in Eq. (4) by exploiting the fact that the longitudinal term depends only on the bandwidth while the transverse ones depend only on the central wavelength. Such a capability allows removing the dependence on the transverse plane and can be useful when the transverse beam size changes during operation.
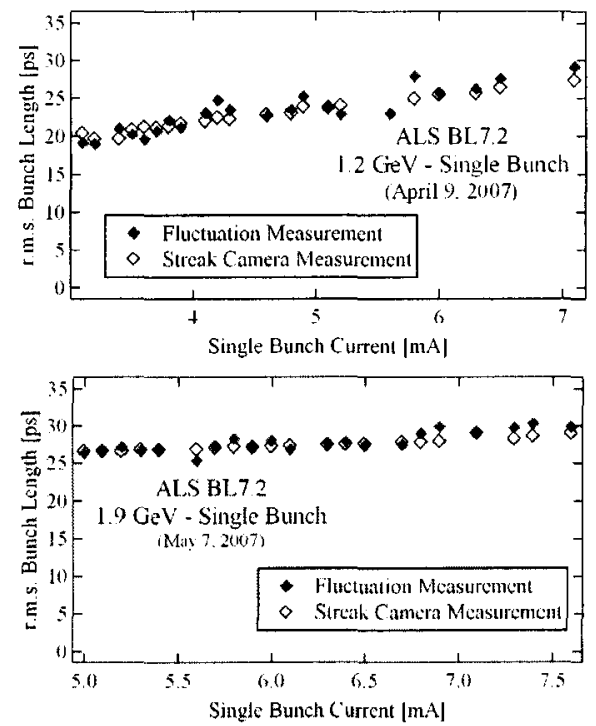

Figure 3. Examples of fluctuation and streak-camera bunch length measurements at the ALS for different beam parameters.

We want also test the system by coupling the light from the source into an optical fiber. This will allow having the measurement setup separated from the source area for an easy accessibility and tuning of the system.

In summary, we have demonstrated an absolute bunch length measurement technique based on the analysis of the fluctuations in the incoherent part of the radiation emitted by a particle beam. The scheme is nondestructive, shows a remarkable simplicity and can be applied in both circular and linear accelerators including cases where the very short length of the bunches makes difficult the use of other techniques.

\section{ACKNOWLEDGEMENTS}

The authors want to thank John Byrd, Stefano De Santis and Josef Frisch for their contributions.

\section{REFERENCES}

[1] M. Zolotorev, G. Stupakov, SLAC PUB 7132 (1996).

[2] P. Catravas et al., Phys. Rev. Letters 82, 5261, (1999).

[3] V. Sajaev, Determination of Longitudinal Bunch Profile using Spectral Fluctuations of Incoherent Radiation, Proceedings of EPAC 2000, Vienna.

[4] M. Yabashi et al., Phys. Rev. Letters 97, 084802 (2006). 